\title{
ВЛИЯНИЕ ОФФШОРИЗАЦИИ НА РЕЗУЛЬТАТЫ ДЕЯТЕЛЬНОСТИ РОССИЙСКИХ ПРЕДПРИЯТИЙ ${ }^{1}$
}

Федорова Е.А. ${ }^{2}$, Николаев А.Э. ${ }^{3}$, Коркмазова Б.К. ${ }^{4}$,

Цель данного исследования - оценка влияния оффшоризация на результаты деятельности отечественных предприятий. В рамках данной цели выдвигалось несколько гипотез.

1. Мы предполагаем, что оффшоры отрицательно влияют на конкурентоспособность российских предприятий и на технологическую цепочку.

2. Оффшоры влияют отрицательно и на экспортно-импортную деятельность России, т.к. искажаются реальные показатели объемов экспорта и импорта страны.

3. Эффект от прямых иностранных инвестиции от стран-оффшоров более выражен, чем эффект от ПИИ развитых стран.

4. В этой гипотезе мы решили оценить, насколько эффективна политика деоффшоризации. Если политика эффективна, то политика деоффшоризации, которую проводит Российская Федерация начиная с 2011 г. должна оказывать положительное влияние на увеличение экспортных и сокращение импортных спилловер-эффектов.

Прямой методологии, с помощью которой мы могли бы провести оценку выдвинутых гипотез, не существует. В нашей работе мы будем использовать методологию, основанную на оценке значимости спилловер-эффектов, или эффектов распространения.

Эмпирическая база включала финансовую отчетность компаний за 2005-2012 гг., была проведена обработка, сортировка и фильтрация данных, после чего осталось 23567 предприятий с долей иностранного капитала и 14653 компаний без него.

В результате исследования было определено, что отрицательное воздействие капитала из оффшорных зон на замкнутую технологическую цепочку неоднозначно, так как существуют положительно направленные страновые спилловер-эффекты. Тенденция приближения страновых показателей к нулю в 2010-2012 гг. говорит о том, что политика деоффшоризации, активно развернувшаяся в 2011 г., уже начала показывать положительные результаты.

Ключевые слова: оффшоры, прямые иностранные инвестиции, спилловер-эффекты, развитые страны, предприятия

\section{JEL:D22, D24, D57}

Иностранные инвестиции на сегодняшний день являются одним из ключевых компонентов мировой экономики, характеризующих развитие как стран в целом, так и отдельных регионов. ПИИ выступают гарантией заинтересованности зарубежных инвесторов в долгосрочном ведении бизнеса на территории принимающего государства, повышая тем самым его экономический потенциал. Россия по итогам последних нескольких лет возглавляет список лидеров по притоку прямых иностранных инвестиций (World Investment Report, 2013). Однако за серьезными цифрами и внешней привлекательностью притока ПИИ скрываются негативные составляющие, в том числе оффшорное происхождение значительной доли притока зарубежного капитала и негативное влияние на конкурентоспособность российских предприятий.

Рассмотрим более подробно оффшоры и их влияние на результаты деятельности отечественных предприятий. Под оффшором (off shore) в современной деловой литературе принято подразумевать полностью или частично свободные от налогов экономические зоны, не предоставляющие информацию о проведенных финансовых операциях. В Докладе о миро-

1. Работа выполнена в рамках прикладной НИР по Государственному заданию Правительства Российской Федерации на 2015 г..

2. Доктор экономических наук, профессор кафедры «Финансовый менеджмент», Финансовый университет при Правительстве Российской Федерации.

3. Стажер-исследователь кафедры «Финансовый менеджмент», Финансовый университет при Правительстве Российской Федерации.

4. Стажер-исследователь кафедры «Финансовый менеджмент», Финансовый университет при Правительстве Российской Федерации 
вых инвестициях, подготовленном ЮНКДАТ в 2013 г., оффшорная природа поступающих в Россию ПИИ выступила примером так называемого «круговорота капитала» (round-tripping) (World Investment Report, 2013, pp. 16, 65). Суть данного явления заключается в том, что иностранный капитал из Кипра или Британских Виргинских островов берет свое начало в России и эти же страны одновременно входят в десятку как крупнейших инвесторов в отечественную экономику, так и реципиентов российских инвестиций (Илюхина, 2013).

Однако открытым остается вопрос, связанный с тем, как влияет оффшорный капитал на результаты деятельности российских предприятий. Политика государства в этом случае является однозначной, государственные лица обозначили оффшоризацию одной из главных экономических проблем современной России, для борьбы с которой с 2013 г. разрабатывается национальный план. Его началом послужило принятие закона Ф3-№134 от 28.06.2013 «О внесении изменений в отдельные законодательные акты Российской Федерации в части противодействия незаконным финансовым операциям» (Федеральный закон Российской Федерации от 28 июня 2013 г. № 134-Ф3), который призван ужесточить государственную финансовую политику, вводя дополнительные меры контроля за оттоком капиталов за рубеж, в оффшорные зоны.

При этом существует полемика в ряде исследований, которая неоднозначно определяет влияние оффшоров на результаты предприятий. Ряд авторов считают, что оффшоризация вредна для экономики в целом и для российской экономики в частности. Такая позиция, сформировалась на основе международных трендов антиоффшорной политики, рассмотренной в статьях Хенри (Henry, 2012), Ка и Фрейтас (Kar and Freitas, 2013), где говорится о колоссальных негативных последствиях для неоффшорных стран от оффшорной деятельности. В отечественной литературе (Андрианов, 2014, 53-59; Костюнина, 2014; Сулакшин, 2013) в качестве негативных аспектов выделяется то, что оффшорные финансовые сети способствуют отмыванию нелегального капитала, недофинансированию национального бюджета, а также использованию оффшорных зон для ухода от налоговых обязательств. Учитывая вышесказанное, позиция государства относительно оффшорных зон вполне предсказуема. Однако ряд авторов придерживаются противоположного мнения. Например, Б. Хейфец (Хейфец, 2008) в своей работе утверждает, что если рассматривать тему оффшоров не с позиции государства в целом, а на примере отдельного предприятия, то всплывают и благоприятные результаты. К таковым можно отнести то, что схемы минимизации налогообложения направлены на повышение конкурентоспособности российских компаний. Также в защиту оффшорных зон приводится наличие независимой судебной системы и достаточно простые и удобные законы для частного предпринимательства. Хейфец (Хейфец, 2008), а также Обухова Е., Огородников Е. (Обухова, Огородников, 2013) говорит, что положительный эффект для отдельного российского предприятия при синергии таких эффектов способен оказаться позитивным и для целого государства

В своей работе мы попытались ответить на вопрос, насколько оффшоры в целом полезны и вредны для российских предприятий. Прямой методологии, с помощью которой мы могли бы провести такую оценку, не существует, в нашей работе мы будем использовать методологию, основанную на оценке значимости спилловер-эффектов, или эффектов распространения.

Ниже представлены сформулированные гипотезы, в которых мы будем предполагать воздействие на результаты деятельности российских предприятий.

Гипотеза 1. В целом по результатам анализа литературы, можно отметить, что в большинстве публикаций доказывается отрицательное воздействие оффшоров на экономику. Поэтому мы предполагаем, что оффшоры отрицательно влияют на конкурентоспособность российских предприятий и на технологическую цепочку. Размеры средств, вложенных в организацию оффшорной зоны, неоправданно большие в сравнении с объемом зарубежных инвестиций, что отрицательно сказывается на взаимодействии предприятий и отраслей одной технологической цепи. 
Проверять данную гипотезу мы будем на основе знаковой направленности спилловер-эффектов от привлечения ПИИ. Данному методу посвящено множество статей мировой экономической литературы ( Aitken, Harrison, Lipsey, 1996; Javorcik, 2004), изучив которые, мы построили свою эмпирическую модель, отражающую российские реалии. Мы использовали классические спилловеры:

Горизонтальный эффект, или эффект вытеснения, рассчитывается по следующей формуле:

HORIZ $_{j t}=\frac{\sum_{i: i \in j, F S_{i j t} \geq 0,25} F S_{i j t} F A_{i j t}}{\sum_{i: i \in j} F A_{i j t}}$

где:

$H O R I Z_{J T}-$ переменная, измеряющая внешний эффект внутри отрасли и представляет собой долю иностранного капитала, инвестированного в компанию в секторе $j$ во время $t$.

$F S_{i j t}$ показывает долю иностранного капитала в фирме $i$ во время $t$ в секторе $j$.

$F A_{i j t}$ показывает стоимость внеоборотных активов фирмы $i$ во время $t$ в секторе $j$.

2. Прямой спилловер-эффект (forward spillover effects) - эффект, передающийся от поставщика к потребителю, который определяется по формуле (2):

$$
F O R W_{\mathrm{jt}}=\beta_{k j, t} H O R I Z_{\mathrm{jt}},
$$

где:

$F R O W_{i t}-$ прямой вертикальный спилловер-эффект для отрасли $j$;

$\beta_{k j, t}$ —доля потребления сектором $k$ продукции, произведенной сектором $j$ за время $t$

3. Обратный спилловер-эффект- эффект, передающийся от потребителя к поставщику, который определяется по формуле (3):

$$
B A C K_{\mathrm{jt}}=\beta_{j k, t} H O R I Z_{\mathrm{jt}}
$$

где:

$B A C K_{j t}$ - обратный вертикальный спилловер-эффект для отрасли $j$;

$\beta_{k j, t}$ - доля выпуска сектора $j$ продукции, потребленного сектором $k$ за время $t$ (Kugler, 2006).

Однако, продолжив работу над темой, мы выявили, что во всех существующих на сегодняшний день формулах спилловер-эффектов используется информация об объемах ПИИ и при этом не принимается во внимание природа их возникновения. Подобный подход приводит к полному игнорированию политической специфики отношений стран - участниц процесса инвестирования, важность которой в своей работе доказали экономисты Лью и и др. (Liu, Daly, Varua, 2012).. Для ликвидации неточности существующих моделей мы вывели на основе стандартных формул горизонтального и вертикального эффектов новый тип спилловеров, отражающих страновой аспект. Таким образом, у нас появилась возможность проверить общепринятые спилловер-эффекты применительно к созданной нами выборке страноффшоров, куда вошли Андорра, Барбадос, Бермуды, Багамские острова, Белиз, Коста-Рика, Кипр, Доминикана, Гренада, Гибралтар, Гонконг, Каймановы острова, Либерия, Монако, Новая Зеландия, Панама, Сейшелы, Сингапур, Сент-Винсент, Британские Виргинские острова.

Горизонтальный страновой спилловер-эффект - горизонтальный спилловер-эффект, определенный для конкретной отрасли, в которой расположена компания или совокупность с иностранным участием из конкретной страны (или стран), рассчитывается по следующей формуле:

HORIZ_str $r_{\mathrm{zjt}}=\frac{\sum_{i, i \in j z, F S_{i, j, t} \geq 0,1} F S_{i, j, t} F A_{i, j, t}}{\sum_{i, i \in j z} F A_{i, j, t}}$,

$\mathrm{HORIZ} \_s t r_{\mathrm{zjt}}-$ горизонтальный эффект для отрасли $j$ от ПИИ из страны $z$. 
По той же аналогии вводим понятия прямого и обратного страновых спилловер-эффектов, определенных для ПИИ из определенной страны:

$$
B A C K_{-} s t r_{\mathrm{zjt}}=\beta_{j k, t} H O R I Z_{-} s t r_{\mathrm{zjt}},
$$

$B A C K_{2} s t r_{\text {zjt }}$ - обратный вертикальный спилловер-эффект для отрасли $j$ от ПИИ из страны $z$.

$$
\text { FORW_str }{ }_{\mathrm{zjt}}=\beta_{k j, t} H_{O R I Z} s t r_{\mathrm{zjt}},
$$

$F O R W_{-} s t r_{\mathrm{zjt}}-$ прямой вертикальный эффект для отрасли $j$ от ПИИ из страны $t$.

Гипотеза 2. Оффшоры влияют отрицательно и на экспортно-импортную деятельность России, т.к. искажаются реальные показатели объемов экспорта и импорта страны.

Механизм оффшоров, как было отмечено ранее, способствует оттоку капитала из национальных экономик и искажает реальные экономические показатели страны. Больше всего страдают инвестиционная активность и показатели экспорта в странах происхождения капитала. Инвестиционная активность снижается, так как в неблагоприятных экономических условиях оффшоры повышают мобильность капитала, который при определенных обстоятельствах мог бы быть реинвестирован в своей стране По такому же принципу происходит занижение показателей объема экспорта, поскольку торговля компании с российскими корнями, функционирующей на территории оффшорной зоны, считается экспортом страны-оффшора, хотя по факту остается отечественным продуктом.

Для того чтобы оценить это влияние, мы вводим понятие импортных и экспортных спилловер-эффектов.

Импортный спилловер-эффект - эффект, передающийся от поставщиков из зарубежных стран к потребителю - резиденту Российской Федерации. Данный эффект определяется по формуле:

IMPORT $_{\mathrm{jt}}=\alpha_{j k, t} \mathrm{HORIZ}_{\mathrm{jt}}$,

$\mathrm{IMPORT}_{\mathrm{jt}}-$ «импортный» спилловер для отрасли ;:

$\alpha_{j k, t}$ - доля потребления российским сектором экономики $j$ за время $t$ продукции, пришедшей из зарубежного сектора экономики $k$.

Таким образом, в случае положительного знака импортного спилловера мы можем судить о том, что компании Российской Федерации сильно зависят от импортных поставщиков. В случае отрицательного знака импортного спилловера мы будем утверждать, что на российской экономике негативно сказывается чрезмерное наличие зарубежных комплектующих. Многим зарубежным компаниям выгоднее открывать предприятия по выпуску комплектующих внутри нашей страны, чем переплачивать за транспортные, таможенные и другие расходы.

Экспортный спилловер-эффект - эффект, передающийся от покупателей из зарубежных стран российским компаниям. Данный эффект определяется по формуле:

$$
\operatorname{EXPORT}_{j t}=\alpha_{k j, t} H_{O R I Z}{ }_{j t}
$$

$E X P O R T_{j t}-$ экспортный спилловер для отрасли $j$;

$\alpha_{k j, t}$ - доля потребления зарубежным сектором экономики $k$ продукции за время $t$, выпущенной российской отраслью $j$.

Аналогично, как и для гипотезы 1, мы сформировали экспортные и импортные спилловеры только для стран-оффшоров.

IMPORT_ $\operatorname{str}_{\mathrm{zjt}}=\alpha_{z j k, t} \mathrm{HORIZ}_{-} \mathrm{str}_{\mathrm{zjt}}$,

IMPORT _ str $_{\mathrm{zjt}}-$ импортный спилловер для отрасли $j$ от ПИИ из страны $z$;

$\alpha_{z k j, t}$ - доля потребления российским сектором экономики $k$ продукции, произведенной сектором $j$ страны $z$ за время $t$. 
EXPORT_str ${ }_{z j t}=\alpha_{z j k, t} H_{\text {ORIZ_ }} s t r_{z j t}$,

$E X P O R T_{-} s t r_{z j t}-$ экспортный спилловер для отрасли $j$ от ПИИ из страны $z$;

$\alpha_{z j k, t}$ - доля потребления отраслью $k$ страны $z$ продукции за время $t$, выпущенной российской отраслью $j$.

Гипотеза 3. Эффект от прямых иностранных инвестиции от стран-оффшоров более выражен, чем эффект от ПИИ развитых стран.

Для того чтобы выявить влияние страны-кредитора на российскую экономику, мы усовершенствовали уже известные нам горизонтальный, вертикальный и обратный эффекты, добавив туда страновой аспект.

Горизонтальный страновой спилловер-эффект - горизонтальный спилловер-эффект, определенный для конкретной отрасли, в которой расположена компания или совокупность с иностранным участием из конкретной страны.

По той же аналогии вводим понятия прямого и обратного страновых спилловер-эффектов и импортный и экспортный спилловер-эффекты для каждой страны. Мы применили данные формулы для стран-оффшоров и сравнили соответствующие эффекты Германии и Франции, так как ПИИ этих стран оказывают наибольшее воздействие на российскую экономику.

Гипотеза 4. В этой гипотезе мы решили оценить, насколько эффективна политика деоффшоризации. Если политика эффективна, то политика деоффшоризации, которую проводит Российская Федерация начиная с 2011 г., должна оказывать положительное влияние на увеличение экспортных и сокращение импортных спилловер-эффектов.

В конце 2011 г. приоритетной задачей для России был объявлен вывод экономики страны из свободных экономических зон. Деоффшоризация - проведение государством комплекса мероприятий в законодательной, правоприменительной и информационной областях для снижения или исключения впоследствии вовлеченности в национальный хозяйственный оборот резидентов под видом иностранных лиц или с использованием иностранных правовых конструкций, преследующих преимущественно незаконные или недобросовестные цели. В рамках данного процесса было выдвинуто множество законопроектов, направленных на усовершенствование системы налогообложения. На данный момент разработан ряд мер, направленных на возведение барьеров на пути отмывания незаконно полученных доходов, а также создаются более выгодные экономические условия для ведения бизнеса в России тем российским компаниям, которые не используют оффшоры в своей деятельности.

При рассмотрении гипотез мы также будем учитывать и временной аспект, поэтому исследования будут проводиться для трех периодов: кризисного, до- и послекризисного. Также для отдельных гипотез будет учтена отраслевая специфика.

\section{Методология исследования}

Все вышеперечисленные гипотезы мы будем проверять с помощью модели, в основе которой лежит производственная функция Кобба-Дугласа.

$$
\begin{aligned}
& \ln \left(Q_{j t}\right)=\beta_{0}+\beta_{1} \ln \left(\text { OSDirect }_{j t}\right)+\beta_{2} \ln \left(\text { TA }_{j t}\right)+\beta_{3} \ln \left(\text { NumEmployees }_{j t}\right)+ \\
& \gamma_{1} \ln \left(\text { HORIZ }_{j t}\right)+\gamma_{2} \ln \left(\text { HORIZ_ }_{-} s r_{z j t}\right)+\gamma_{3} \ln \left(\text { BACK }_{j t}\right)+\gamma_{4} \ln \left(\text { BACK_str }_{z j t}\right)+ \\
& \gamma_{5} \ln \left(\text { FORW }_{j t}\right)+\gamma_{6} \ln \left(\text { FORW_ }_{-} s t r_{z j t}\right)+\eta_{1} \ln \left(\text { IMPORT }_{j t}\right)+\eta_{2} \ln \left(\text { IMPORT_str }_{z j t}\right)+ \\
& \eta_{3} \ln \left(\text { EXPORT }_{j t}\right)+\eta_{4} \ln \left(\text { EXPORT_str }_{z j t}\right),
\end{aligned}
$$

где:

$i$ - индекс компании;

$j, k$ индексы отрасли; 
$z$ - индекс страны;

$Q_{i t}$ - общая выручка $i$-компании;

OSDirect $_{j t}=F S_{i}$ - доля иностранного капитала в фирме $i$;

$T A_{j t}$ - объем активов компаний в отрасли $j$ во время $t$;

NumEmployees $_{\text {it }}$ - количество персонала $i$-й компании во время $t$.

Все остальные переменные описаны выше в гипотезах.

\section{Данные}

В ходе работы использовалась база российских компаний за 2005-2012 гг., взятая из информационного ресурса RusLana, который содержит в себе информацию о балансовых показателях, прибыли и убытках предприятий, структуре капитала, количестве занятых сотрудников, а также отраслевой принадлежности предприятия. Данные были отобраны по критериям:

1. Организационно правовая форма предприятия: ОАО, ЗАО, АООТ.

2. Количество работников на предприятии - от 50 человек.

3. Доля иностранного капитала свыше $10 \%$. В соответствии с третьим критерием компании разделились на фирмы с ПИИ и без. Была проведена обработка, сортировка и фильтрация данных, после чего осталось 23567 предприятий с долей иностранного капитала и 14653 компаний без него.

\section{Результаты}

На следующем этапе анализа проверим выдвинутые гипотезы для разных временных периодов. Нас интересует не только влияние офшорных зон на состояние экономики России, но и динамика этого влияния в относительно кризисные и благополучные периоды. В таблице 1 представлены результаты расчетов вертикальных и горизонтальных и страновых спилловерэффектов.

Таблица 1

Оценка влияния оффшоров на выручку российских предприятий

\begin{tabular}{|c|c|c|c|c|}
\hline \multirow[t]{2}{*}{ Показатели } & \multicolumn{4}{|c|}{ Значимость } \\
\hline & 2005-2007 & 2008-2009 & 2010-2012 & 2005-2012 \\
\hline Const & $-1,081 * * *$ & $-2,383 * * *$ & $0,278 * * *$ & $-0,959 * * *$ \\
\hline OSDirect & $-0,037 * * *$ & $-0,020 * * *$ & $-0,018 * * *$ & $-0,024 * * *$ \\
\hline TA & $0,990 * * *$ & $1,024 * * *$ & $0,956^{* * *}$ & $0,986^{* * *}$ \\
\hline NumEmployees & $0,302 * * *$ & $0,499 * * *$ & $0,164 * * *$ & $0,313 * * *$ \\
\hline HORIZ_X & $-0,402 * * *$ & $-0,344 * * *$ & $-0,011$ & $-0,259 * * *$ \\
\hline HORIZ..оффшор & $-0,992 * * *$ & $-0,958 * * *$ & $-0,163 * *$ & $-0,162 * *$ \\
\hline BACK_X & $0,917 * * *$ & $0,532 * * *$ & $0,845^{* * *}$ & $0,704 * * *$ \\
\hline Back.str_oффшор & $0,917 * * *$ & $0,326^{* * *}$ & $0,125^{*}$ & $0,704 * * *$ \\
\hline FORWARD_X & $-0,785^{* * *}$ & $-0,369 * * *$ & $-0,838 * * *$ & $-0,605^{* * *}$ \\
\hline Forward.str_оффшор & $-0,372 * * *$ & $-0,400 * * *$ & $-0,027$ & $-0,116^{*}$ \\
\hline Import_X & $0,061 * * *$ & $0,062 * * *$ & $0,031^{* *}$ & $0,045^{* * *}$ \\
\hline Import.str_оффшор & $-0,131 * * *$ & $-0,157 * * *$ & $-0,111 * * *$ & $-0,122 * * *$ \\
\hline Export_X & 0,011 & 0,028 & 0,020 & $0,026^{* * *}$ \\
\hline Export_X.оффшор & $0,108 * * *$ & $0,140 * * *$ & $0,088 * * *$ & $0,096^{* * *}$ \\
\hline
\end{tabular}

Примечание. Значимость оченивалась по z-статистике. Критические значения z-статистик при уровнях значимости: $*-10 \% ; * *-5 \% ; * * *-1 \%$.

Как видно из данных таблицы 1, практически во всех случаях коэффициенты значимости, за исключением экспортного спилловера, стремятся к нулю. Это означает, что модель ста- 
тистически значима. Иностранные инвестиции, поступая в различные компании, вызывают не только вертикальные и горизонтальные спилловер- эффекты, но также импортные и экспортно-страновые спиловеры. При этом если прямые вертикальные и горизонтальные спилловеры отрицательно направлены, то обратные вертикальные, экспортные и импортные спилловер-эффекты оказывают положительное влияние на состояние отечественных компаний. Горизонтальные и вертикальные эффекты по России в целом, не принимая в расчет направленность знака, обладают гораздо большим влиянием, чем экспортные и импортные спилловеры. Рассмотрим подробнее нововведенные страновые эффеты

Горизонтальный оффшорный спилловер-эффект отражает влияние горизонтального спиловера, возникшего в результате притока ПИИ от стран-оффшоров. По результатам анализа он отрицательный на всех трех временных промежутках, но при этом имеет тенденцию к снижению модульного значения с 0,402 до 0,011 , т.е фактически до 0. Это говорит о том, что появление в отраслях оффшорного иностранного капитала имеет негативный характер и ухудшает итоговые показатели деятельности предприятий в отраслях. Достаточно резкое снижения модульного значения в период 2010-2012 гг. говорит о «привыкании» экономики к оффшорным инвестициям, за счет чего снижается подверженность их отрицательному влиянию, а также сказывается политика деоффшоризации, выбранная правительством России и набравшая к 2011 г. особенно сильные обороты. Однако при этом негативный эффект оффшоризации гораздо сильнее, чем эффект от остальных иностранных инвестиций.

Прямой вертикальный оффшорный спилловер-эффект показывает слабую волатильность и тенденцию снижения модульного значения к нулю. Устойчивое отрицательное влияние на потребителей фирм с иностранным капиталом в начале XXI в. и его постепенное приближение к нулю, как и в случае с горизонтальным страновым эффектом, подтверждает как нашу гипотезу о вреде ПИИ от стран-оффшоров, так и наше предположение о том, что политика деоффшоризации корректирует негативное воздействие притока оффшорного капитала.

Обратный вертикальный оффшорный спилловер-эффект по силе воздействия самый значимый и единственный положительный среди оффшорных эффектов. Однако, как и вышеописанные результаты, постепенно с 0,917 в 2005-2007 гг. снижается до 0,125 к 2012 г. Это говорит о том, что российские компании научились взаимодействовать друг с другом и их прочные связи не подвергаются негативному влиянию оффшоров, они используют любое влияние капитала для минимизации издержки за счет развития своих партнеров и системы логистики. Этот эффект можно также объяснить тем, что целью оффшоризации является уход от налогов, компании являются теми же самыми, и поэтому они менее воздействуют на поставщиков, не передавая им знания и умения, как остальные компании с ПИИ.

Импортный оффшорный эффект обладает отрицательной знаковой направленностью. Это происходит потому, что импорт из стран-оффшоров по сути своей означает покупку у отечественной компании, с увеличением издержек, связанных с доставкой товара из одной страны в другую. При этом происходит завышение показателей импорта страны, что не может сказываться на экономике страны положительно.

Экспортный оффшорный эффект стабильно положителен и обладает средней магнитудой. Так же как в случае с импортом, при экспорте в страны-оффшоры происходит искажение реальных показателей, поэтому положительное воздействие экспортного оффшорного эффекта достаточно условно. Однако подобные результаты подтверждают нашу гипотезу о том, что политика деоффшоризации, которую проводит Российская Федерация начиная с 2011 г., оказывает положительное влияние на увеличение экспортных и сокращения импортных спилловер-эффектов. Судя по значимости экспортного эффекта, экспортоориентированные компании более всего являются оффшоризованными.

Далее рассмотрим страновые эффекты оффшорных стран в сравнении с соответствующими эффектами развитых стран - Германии и Франции. 
Оценка влияния прямых иностранных инвестиций от стран оффшоров и развитых стран

\begin{tabular}{|c|c|c|c|}
\hline Показатели & \multicolumn{2}{|c|}{ Значимость } & Оффшоры \\
\hline & Германия & Франция & $-0,959^{* * *}$ \\
\hline Const & $-0,958^{* * *}$ & $-0,959^{* * *}$ & $-0,024^{* * *}$ \\
\hline x1-OSDirect & $-0,026^{* * *}$ & $-0,026^{* * *}$ & $0,986^{* * *}$ \\
\hline x2-TA & $0,985^{* * *}$ & $0,985^{* * *}$ & $0,313^{* * *}$ \\
\hline x3-NumEmployees & $0,315^{* * *}$ & $0,985^{* * *}$ & $-0,162^{* *}$ \\
\hline x5-HORIZ_cтрана & $0,207^{* *}$ & $0,299^{*}$ & $0,704^{* * *}$ \\
\hline x7-Back_cтрана & $0,128^{*}$ & $0,119^{*}$ & $-0,116^{* * *}$ \\
\hline x9-Forward_cтрана & $-0,280^{*}$ & $-0,202^{*}$ & $-0,122^{*}$ \\
\hline x11-Import_cтрана & $-0,066^{* *}$ & $0,081^{* *}$ & $0,096^{* * *}$ \\
\hline x13-Export_cтрана & $0,097^{* * *}$ & $-0,090^{* *}$ & \\
\hline
\end{tabular}

Выдвинутая нами гипотеза о том, что степень влияния страновых спилловер-эффектов выше у стран-оффшоров, чем у развитых стран, лишь частично подтвердилась результатами из таблицы 2.

Обратный вертикальный и импортный страновые эффекты действительно выше для страноффшоров, чем для Франции или Германии; при этом обратный эффект несет в себе положительную направленность, что говорит о большем положительном эффекте от сотрудничества со странами-оффшорами в сравнении с развитыми странами.

Горизонтальный и прямой вертикальный страновые эффекты, напротив, выше для Германии и Франции, при этом положительное воздействие горизонтального эффекта от развитых стран способно перекрыть негативные последствия от притока оффшорного капитала. Горизонтальный спилловер-эффект для стран-оффшоров является отрицательным, потому что компании, которые зарегистрированы как оффшорные, обладают более высокой конкурентоспособстью из-за налоговых льгот.

На подобном противоречии разных показателей полностью подтвердить или опровергнуть гипотезу о большей степени влияния страновых эффектов от оффшорных зон достаточно сложно. Однако при правильной комбинации потоков из различных стран можно нейтрализовать негативные эффекты и умножить благоприятное воздействие.

Исследования отечественной (Кузнецов, 2012a; Либман и Хейфец, 2007) и зарубежной литературы (Kalotay, 2012; Rugman and Verbeke, 2004) по вопросу оффшоров, где, с одной стороны, описывается неоспоримый вред оффшоров, связанный с отмыванием нелегального капитала и уходом от налогов, а с другой - говорится, что наличие оффшоров и создаваемая ими налоговая конкуренция сдерживают растущий уровень налогов, а также наши собственные результаты не позволяют прийти к однозначному пониманию характера влияния оффшорных зон на российскую экономику. Поэтому мы решили рассмотреть ситуацию еще под одним углом. Проверить, как себя ведут рассматриваемые нами спилловеры, на примере трех самых значимых для отечественной экономики отраслей - торговой, производственной и добывающей. Результаты исследования представлены в таблице 3.

Оценка отраслевой специфики

\begin{tabular}{|c|c|c|c|}
\hline Показатели & \multicolumn{3}{|c|}{ Значимость } \\
\hline & Торговля & Производство & Добывающая отрасль \\
\hline Const & $-1,081$ & $-0,933^{* * *}$ & $-1,462 * * *$ \\
\hline OSDirect & $-0,025^{* * *}$ & $-0,005$ & $-0,053^{* * *}$ \\
\hline TA & $0,987 * * *$ & $1,026 * * *$ & $0,954 * * *$ \\
\hline NumEmployees & $0,362 * * *$ & $0,166^{* * *}$ & $0,352^{* * *}$ \\
\hline HORIZ_X & $0,159 *$ & 0,122 & $-0,817^{* * *}$ \\
\hline
\end{tabular}




\begin{tabular}{|c|c|c|c|}
\hline HORIZ..otr.str._X & $-0,405^{* * *}$ & $0,766^{*}$ & $0,527^{*}$ \\
\hline BACK_X & $-0,211^{* * *}$ & $-0,206^{* *}$ & $0,878^{* * *}$ \\
\hline Back.str_X & $-0,516^{* * *}$ & 0,077 & $-2,090^{* * *}$ \\
\hline FORWARD_X & $0,206^{* * *}$ & $-0,040$ & $-0,096$ \\
\hline Forward.str_X & $0,898^{* * *}$ & 0,043 & $1,308^{* * *}$ \\
\hline Import_X & $0,087^{* * *}$ & $-0,100^{* * *}$ & $-0,004$ \\
\hline Import.str_X & 0,032 & 0,107 & 0,011 \\
\hline Export_X & $-0,020$ & $0,155^{* * *}$ & $0,072^{*}$ \\
\hline Export_X.str & $-0,049$ & $-0,143^{*}$ & $-0,103$ \\
\hline
\end{tabular}

Интересно отметить, что произошло снижение значимости некоторых оффшорных эффектов. Так, обратный оффшорный эффект, оказывая сильное отрицательное воздействие на предприятия торговой и добывающей отрасли, оказался абсолютно незначимым для производящей. Аналогичная ситуация и с прямым вертикальным оффшорным эффектом, который в случае с добывающей отраслью показывает сильное положительно направленное воздействие, что подтверждает наши сомнения об исключительно негативной природе оффшоров.

Что касается импортного и экспортного спилловеров, можно отметить, что предприятия добывающей отрасли, несмотря на то что ее принято считать экспорториентированной, практически не зависят ни от объемов импорта или экспорта, ни от того, с какой страной осуществляется сделка. В это же время производящие компании подвергаются отрицательному воздействию импортного спилловера и положительному - экспортного. В целом из таблицы видно, что чувствительней всего на какое-либо сотрудничество с компаниями из оффшорных зон реагируют компании добывающей отрасли. Торговля, производство и добывающая отрасли оказались наиболее подвержены влиянию страновых и оффшорных спилловер-эффектов, в то время как здравоохранение и образование, для которых мы также проводили исследование, показали себя вполне устойчивыми к изменению в структуре источников ПИИ, а также к экспортно-импортным отношениям.

\section{Общие выводы по работе}

Иностранные инвестиции, поступая в различные компании, вызывают не только вертикальные и горизонтальные спилловер-эффекты, но также импортные и экспортные спилловеры. При этом, если прямые вертикальные и горизонтальные спилловеры отрицательно направлены, то обратные вертикальные, экспортные и импортные спилловер-эффекты оказывают положительное влияние на состояние отечественных компаний.

Отрицательное воздействие капитала из оффшорных зон на замкнутую технологическую цепочку неоднозначно, так как существуют положительно направленные страновые спилловерэффекты.

Из-за противоречия результатов полностью подтвердить или опровергнуть гипотезу о том, что степень влияния страновых спилловер-эффектов выше у стран-оффшоров, чем у развитых стран, достаточно сложно. Однако при правильной комбинации потоков из различных стран можно нейтрализовать негативные эффекты и умножать благоприятное воздействие.

Тенденция приближения страновых показателей к нулю в 2010-2012 гг. говорит о том, что политика деоффшоризации, активно развернувшаяся в 2011 г., уже начала показывать положительные результаты.

\section{Список литературы}

1. Андрианов В., Кривопустова Е. Офшоры и деоффшоризация экономики// Общество и экономика. 2014. № 5. С. 53-91.

2. Илюхина Е. Иностранные инвестиции в һоссийской экономике // Экономическое развитие России, 2013. №10. URL: http://www.iep.ru/files/RePEc/gai/ruserr/81Ilyuhina.pdf. 
3. Либман А., Хейфец Б. Корпоративная модель региональной экономической интеграции // Мировая экономика и международные отношения. 2007. № 3. С. 15-22.

4. Обухов Е., Огородников Е. Пора возвращаться с островов // Эксперт. 2013. № 4.

5. Офшорный бизнес во внешнеэкономической деятельности. Учеб. Пособие / Под ред. Костюниной Г.М. М.: ИНФРА-М, 2014. Кузнецов А.В. Российские прямые инвестиции в странах СНГ // Международные процессы. 2013. № 2. С. 18-28.

6. Сулакшин С.С. и др. Отток капитала из России: проблемы и решения // Труды Центра проблемного анализа и государственно-управленческого проектирования. Вып. 26. М.: Научный эксперт, 2013

7. Хейфец Б.А. Офшорные юрисдикции в глобальной и национальной экономике. М.: Экономика, 2008.

8. Федеральный закон Российской Федерации от 28 июня 2013 г. № 134-Ф3 г. URL: http:// www.rg.ru/2013/07/02/finansi-dok.html.

9. Aitken, B., Harrison, A., and Lipsey, R. (1996), "Wages and Foreign Ownership: A Comparative Study of Mexico, Venezuela, and the United States", Journal of International Economics, no. 40, pp. 345-371.

10. Henry, J. (2012), "The Price of Offshore Revisited”, Tax Justice Network. July.

11. Javorcik, B.S. (2004), "Does Foreign Direct Investment Increase the Productivity of Domestic Firms? In Search of Spillovers through Backward Linkages", American Economic Review, vol. 94, no. 3, pp. 605-627.

12. Kalotay, K. (2012), “Indirect FDI”, Journal of World Investment \& Trade, vol. 13, no 4, pp. $542-555$.

13. Kar, D., and Freitas, S. (2013), "Russia: Illicit Financial Flows and the Role of the Underground Economy"m Global Financial Integrity.

14. Kugler, M. (2006), "Spillovers from Foreign Direct Investment: Within or between Industries?", Journal of Development Economics, vol. 88, no. 2, pp. 444-477.

15. Liu, K., Daly, K., and Varua, M.E. (2012), “Determinants of Regional Distribution of FDI I nflows across China's Four Regions”, International Business Research, vol. 5, no. 12, pp. $119-126$.

16. Rugman, A.M., and Verbeke, A. (2004), “A Perspective on Regional and Global Strategies of Multinational Enterprises", Journal of International Business Studies, vol. 35, no 1,pp.. 3-18.

17. World Investment Report 2013: Global Value Chains: Investment and Trade for Development . UNCTAD, 2013.

18. World Investment Report 2013: Global Value Chains: Investment and Trade for Development. UNCTAD, 2013. Pp.16, 65/ 


\title{
THE IMPACT OF OFFSHORE RESULTS \\ OF RUSSIAN ENTERPRISES
}

\author{
Fedorova E.A., \\ Professor of Financial Management chair, \\ Financial University under the Government of the Russian Federation \\ Nikolaev A.E., \\ Financial Management chair, \\ Financial University under the Government of the Russian Federation.
}

Korkmazova B. K.,

Financial Management chair,

Financial University under the Government of the Russian Federation.

\begin{abstract}
This paper evaluates influence of the offshore on domestic companies. It is based on the following hypotheses:

The offshores negatively affect the competitiveness of Russian enterprises and the processing chain The offshores negatively affect export-import activities in Russia, as they distort the real figures of country's exports and imports

The effect of foreign direct investment from offshore countries is more pronounced than the effect of FDI in developed countries

We decided to evaluate the effectiveness of deoffshorization policy. If deoffshorization policy pursued by the Russian Federation since 2011 is effective, it should have a positive impact on the export's increase and import spillover effects' decrease.

Where is no direct methodology we could assess our hypotheses. In our work, we used a methodology based on an assessment of spillover effects.

The study was based on statements of Russian companies (23 567 joint enterprises and 14653 companies without FDI) for the period of 2005-2012. The authors used RusLana resource to get following information: balance sheets, profit and loss statements, capital structure, number of employees and regional affiliation.

The study found that the negative impact of capital from offshore zones to the closed process chain is ambiguous, as there are positive country spillover effects. The trend approaching country indicators to zero in 2010-2012. It suggests that the deoffshorization policy already has showed positive results.
\end{abstract}

Key words: offshores, foreign direct investment, Industry-specific, spillover effect, developed country, enterprise

\section{Referenses}

1. Aitken B., Harrison A., Lipsey R. (1996.) Wages and Foreign Ownership: A Comparative Study of Mexico, Venezuela, and the United States. Journal of International Economics, Vol. 40, pp. 345-371.

2. Henry J. (2012). The Price of Offshore Revisited / Tax Justice Network. July

3. http://expert.ru/dossier/story/deofshorizatsiya/ EHkspert-Onlajn.

4. http://www.iep.ru/files/RePEc/gai/ruserr/81Ilyuhina.pdf E. Ilyuhina Inostrannye investicii v Rossijskoj ehkonomike. //EHkonomicheskoe razvitie Rossii, 2013. №10

5. Javorcik B. S. (2004). Does Foreign Direct Investment Increase the Productivity of Domestic Firms? In Search of Spillovers through Backward Linkages. American Economic Review, Vol. 94, No. 3, pp. 605-627. 
6. Kalotay K. (2012). Indirect FDI // Journal of World Investment \& Trade. Vol. 13, No 4. P. 542-555.

7. Kar D., Freitas S. (2013). Russia: Illicit Financial Flows and the Role of the Underground Economy / Global Financial Integrity. February.

8. Kugler M. (2006). Spillovers from Foreign Direct Investment: Within or between Industries? Journal of Development Economics, Vol. 88, No. 2, pp. 444-477.

9. Liu K., Daly K., Varua M.E. Determinants of Regional Distribution of FDI Inflows across China's Four Regions // International Business Research. - Dec 2012, Vol. 5, Issue 12, p 119-126.

10. Rugman A. M., Verbeke A. (2004). A Perspective on Regional and Global Strategies of Multinational Enterprises // Journal of International Business Studies. Vol. 35, No 1. P. 3-18.

11. World Investment Report 2013: Global Value Chains: Investment and Trade for Development // UNCTAD, 2013

12. World Investment Report 2013: Global Value Chains: Investment and Trade for Development // UNCTAD, 2013. P.16, P.65

13. Kostyunina G. M. (red.) (2014). Ofshornyj biznes vo vneshneehkonomicheskoj deyatel'nosti. Ucheb. posobie. M.: INFRA-M. [Kostyunina G. M. (ed.) (2014). Offshore Business in Foreign-Economic Activity. A Textbook. Moscow: INFRA-M. (In Russian).]

14. Kuznecov A. V. (2012a). Rossijskie pryamye investicii v stranah SNG // Mezhdunarodnye processy. N 2. S. 18-28. [Kuznetsov A. V. (2012a). Russian Foreign Investments in the CIS countries // Mezhdunarodnye Protsessy. No 2. P. 18-28.]

15. Libman A., Hejfec B. (2007). Korporativnaya model' regional'noj ehkonomicheskoj integracii // Mirovaya ehkonomika i mezhdunarodnye otnosheniya. N 3. S. 15-22. [Libman A., Kheyfets B. (2007). Corporate Model of Regional Economic Integration // Mirovaya Ekonomika i Mezhdunarodniye Otnosheniya. No 3. P. 15-22.]

16. Ofshory i deofshorizaciya ehkonomiki. V. Andrianov, E. Krivopustova, Obshchestvo $i$ ehkonomika, № 5, Maj 2014, C. 53-91

17. Pora vozvrashchat'sya s ostrovov // EHkspert. N 4. [Obukhov E., Ogorodnikov E. (2013). It's Time to Get Back from the Islands // Expert. No 4.]

18. Sulakshin S. S. i dr. (2013). Ottok kapitala iz Rossii: problemy i resheniya // Trudy Centra problemnogo analiza i gosudarstvenno-upravlencheskogo proektirovaniya. Vyp. 26. M.: Nauchnyj ehkspert. [Sulakshin S. S. et al. (2013). Capital Flow from Russia: Issues and Decisions. Proceedings of the Center for Problem Analysis and State and Managerial Planning, Issue 26. (In Russian).

19. Federal'nyj zakon Rossijskoj Federacii ot 28 iyunya 2013 g. N 134-FZ g. Moskva http:// www.rg.ru/2013/07/02/finansi-dok.html

20. Hejfec B. A. (2008). Ofshornye yurisdikcii v global'noj i nacional'noj ehkonomike. M.: EHkonomika. [Kheyfets B. A. (2008). Offshore Jurisdictions in Global and National Economy. Moscow: Ekonomika.] 